\title{
A flyby anomaly for Juno? Not from standard physics
}

\author{
L. Iorio \\ Ministero dell'Istruzione, dell'Università e della Ricerca (M.I.U.R.)-Istruzione \\ Fellow of the Royal Astronomical Society (F.R.A.S.) \\ Viale Unità di Italia 68, 70125, Bari (BA), Italy \\ Tel. +39 329 2399167. Fax: N/A. email: lorenzo.iorio@libero.it
}

August 24, 2018

\begin{abstract}
An empirical formula recently appeared in the literature to explain the observed anomalies of about $\Delta \dot{\rho} \approx 1-10 \mathrm{~mm} \mathrm{~s}^{-1}$ in the geocentric range-rates $\dot{\rho}$ of the Galileo, NEAR and Rosetta spacecraft at some of their past perigee passages along unbound, hyperbolic trajectories. It predicts an anomaly of the order of $6 \mathrm{~mm} \mathrm{~s}^{-1}$ for the recent flyby of Juno, occurred on 9 October 2013. Data analyses to confirm or disproof it are currently ongoing. We numerically calculate the impact on the geocentric Juno's range rate of some classical and general relativistic dynamical effects which are either unmodelled or mismodelled to a certain level in the software used to process the data. They are: a) The first even zonal harmonic coefficient $J_{2}$ of the multipolar expansion of the terrestrial gravitational potential causing orbital perturbations both at the $\left.\mathrm{a}^{\prime}\right)$ Newtonian $\left(J_{2}\right)$ and at the $\left.\mathrm{a}^{\prime \prime}\right)$ first post-Newtonian level $\left(J_{2} c^{-2}\right)$ b) The post-Newtonian gravitoelectric (GE) Schwarschild-like component of the Earth's gravitational field c) The post-Newtonian gravitomagnetic (GM) Lense-Thirring effect. The magnitudes of their mismodeled and nominal range-rate signatures are: $\left.\left.\mathrm{a}^{\prime}\right) \Delta \dot{\rho}_{\sigma_{J_{2}}} \approx 1 \mu \mathrm{m} \mathrm{s}^{-1} \mathrm{a}^{\prime \prime}\right) \Delta \dot{\rho}_{J_{2} c^{-2}} \approx 0.015 \mu \mathrm{m} \mathrm{s}^{-1}$ b) $\Delta \dot{\rho}_{\mathrm{GE}} \approx 25$ $\mu \mathrm{m} \mathrm{s}^{-1}$ c) $\Delta \dot{\rho}_{\mathrm{GM}} \approx 0.05 \mu \mathrm{m} \mathrm{s}^{-1}$. If a flyby anomaly as large as a few $\mathrm{mm} \mathrm{s}^{-1}$ will be finally found also for Juno, it will not be due to any of these standard gravitational effects. It turns out that a Rindlertype radial extra-acceleration of the same magnitude as in the Pioneer anomaly would impact the Juno's range-rate at a $\Delta \dot{\rho}_{\text {Rin }} \approx 1.5 \mu \mathrm{m} \mathrm{s}^{-1}$ level. Regardless of the quest for the flyby anomaly, all such effects are undetectable.
\end{abstract}


Keywords:

Experimental studies of gravity;

Experimental tests of gravitational theories;

Modified theories of gravity;

Lunar, planetary, and deep-space probes

PACS:

04.80.-y;

04.80.Cc;

04.50.Kd;

95.55.Pe

\section{Introduction}

On 9 October 2013, the NASA's spacecraft Juno ${ }^{1}$ [1] made an Earth flyby passing to within $561 \mathrm{~km}$ of our planet at $19: 21$ GMT to gain the required gravitational energy to reach Jupiter, its final target, in July 2016 [2].

Such an event raised interest [3-6] because of its potential capability to shed more light on one of the recently reported astrometric anomalies in the Solar System [7]: the so-called flyby anomaly [8-12]. It consists of a small, unexpected increase of the geocentric range-rate

$$
\Delta \dot{\rho} \approx 1-10 \mathrm{~mm} \mathrm{~s}^{-1}
$$

experienced by some spacecraft (Galileo, NEAR, Rosetta) approaching the Earth along unbound, hyperbolic trajectories in occasion of some of their flybys. At present, no satisfactory explanations exist for such a phenomenon in terms of both conventional gravitational and non-gravitational physics; see, e.g., [12,13] and references therein. In particular, in [14] it was shown that the thermal effects which should be responsible for most of the Pioneer anomaly [15] could not explain the Rosetta flyby anomaly. Possible spacecraft electrostatic charging effects in terms of a Lorentz force were ruled out in [16]. For the-negligible-impact of the general relativistic gravitomagnetic Lense-Thirring effect on the motion of a test particle in hyperbolic motion, see $[17,18]$. Another negative result in term of the Kerr geometry in the context of Conformal Gravity was recently obtained in [19]. Several more or less sound explanations in terms of non-conventional physics have been put forth so far [20-42] with mixed success. We mention also a proposed modification of inertia [43,44], and the effect of Earth-bound Dark Matter [45-48].

\footnotetext{
${ }^{1}$ See also http://missionjuno.swri.edu/ and http://www.nasa.gov/ mission_pages/juno/ on the Internet.
} 
Proposals have been made to test the flyby anomaly with dedicated future space-based missions [49-51].

The opportunity offered by Juno is, in principle, interesting also because of the relatively low altitude of its flyby of Earth. The expected effect is of the order of $[5]^{2}$

$$
\Delta \dot{\rho}_{\text {Juno }} \approx 7 \mathrm{~mm} \mathrm{~s}^{-1} .
$$

The figure in eq. (2) can be obtained, e.g., by using the empirical formula devised in [10] to accommodate some of the previously observed flybys of the other spacecraft. However, it should be recalled that the formula by Anderson et al. [10] gives wrong (not null) anomaly predictions for the second and third Rosetta flybys. In waiting for the final outcome of the ongoing analysis by NASA/JPL of the data collected by ESA aimed to establish if the flyby anomaly exists also for Juno or not, in this paper we will quantitatively look at the effects of some standard Newtonian/Einsteinian gravitational effects on the geocentric range-rate of the Jupiter-targeted spacecraft at the epoch of its terrestrial flyby. Some of them, like the Lense-Thirring effect, recently detected in the Earth's gravitational field with a claimed 19\% accuracy [52], are unmodeled in the softwares used to process the spacecraft's data, while others are modeled with a necessarily limited accuracy. Our aim is to calculate the size of such range-rate signals to see if they are relevant at a $\sigma_{\dot{\rho}} \approx \mathrm{mm} \mathrm{s}^{-1}$ level of accuracy and, in particular, if they could allow for an effect as large as eq. (2).

\section{Numerical simulations}

In order to investigate the impact of some gravitational effects which, in principle, may induce a flyby anomaly for Juno, we numerically integrate its equations of motion in a geocentric reference frame with Cartesian orthogonal coordinates. For each additional acceleration $\boldsymbol{A}_{\text {pert }}$ with respect to the Newtonian monopole $\boldsymbol{A}_{\mathrm{N}}$, viewed as a small perturbation of it, we perform two numerical integrations: one in which the total acceleration is $\boldsymbol{A}_{\text {tot }}=\boldsymbol{A}_{\mathrm{N}}+\boldsymbol{A}_{\text {pert }}$, and one in which we keep only $\boldsymbol{A}_{\mathrm{N}}$. Both the integrations share the same initial conditions, retrieved from the HORIZONS WEB interface by NASA/JPL. Then, from the resulting time series $\left\{x_{\text {pert }}(t), y_{\text {pert }}(t), z_{\text {pert }}(t)\right\}$ and $\left\{x_{\mathrm{N}}(t), y_{\mathrm{N}}(t), z_{\mathrm{N}}(t)\right\}$ for the geocentric coordinates we produce two time series $\dot{\rho}_{\text {pert }}(t)$ and $\rho_{\mathrm{N}}(t)$ for the range-rate $\rho$ and take, their difference to obtain $\Delta \dot{\rho}$ which singles out the expected signature

\footnotetext{
${ }^{2}$ It may interesting to note that an anomaly with the same magnitude but with the opposite sign was predicted in [6] .
} 
of the effect one is interested in on the Juno's range rate. The integration time span is $\Delta t=4000 \mathrm{~s}$, starting from the shadow entry, so that the flyby occurs after $1260 \mathrm{~s}$ from $t_{0}=0$.

In Figure 1 we depicts our results for the following dynamical features.

- The Newtonian effect of the Earth's oblateness, parameterized by the first even zonal harmonic $J_{2}=-\sqrt{5} \bar{C}_{2,0}$, where $\bar{C}_{\ell, m}$ are the normalized Stokes coefficients of degree $\ell$ and order $m$ of the geopotential [53]. In the left upper corner, its nominal range-rate shift is depicted. It shows a peak-to-peak amplitude of a few $\mathrm{m} \mathrm{s}^{-1}$. Actually, global Earth's gravity field models are usually adopted in the data reduction softwares, so that one has to look just at the residual range-rate signature left by the unavoidable mismodeling in $J_{2}$ as a potential cause for a flyby anomaly. By conservatively evaluating $\sigma_{\bar{C}_{2,0}}$ as in [54] on the basis of the independent approach recently put forth in [55], we obtained the signal displayed in the right upper corner of Figure 1. Its amplitude is as little as $1 \mu \mathrm{m} \mathrm{s}^{-1}$.

- The first post-Newtonian (1PN) gravitoelectric (GE), Schwarzschildlike component of the Earth's field, usually modeled in the data analysis softwares. The left mid panel shows its nominal range-rate signature, which amounts to $25 \mu \mathrm{m} \mathrm{s}^{-1}$.

- The first post-Newtonian (1PN) gravitomagnetic (GM), Lense-Thirring component of the Earth's field, usually unmodeled in the data analysis softwares. The peak-to-peak amplitude of its signal, shown in the right mid panel of Figure 1, is as little as $0.05 \mu \mathrm{m} \mathrm{s}^{-1}$.

- The first post-Newtonian (1PN) aspherical component of the Earth's field, proportional to $J_{2} c^{-2}$ [56-58], usually unmodeled in the data analysis softwares. Its effect on the Juno's range-rate, displayed in the left lower corner of Figure 1, amounts to $0.015 \mu \mathrm{m} \mathrm{s}^{-1}$.

- A Rindler-type radial acceleration [59-61] with the same magnitude as in the Pioneer anomaly (right lower corner). As elucidated in [60,61], it may not be applicable to huge bodies of astronomical size, contrary to man-made objects such as spacecraft like, e.g., the Pioneer probes. Indeed for a body of mass $m_{\mathrm{b}}$ and size $d_{\mathrm{b}}$, the condition $[60,61]$

$$
\frac{G m_{\mathrm{b}}}{d_{\mathrm{b}}} \lesssim\left|A_{\text {Rin }}\right| r
$$


must be satisfied. Since ${ }^{3}[1] m_{\mathrm{J}}=3625 \mathrm{~kg}, d_{\mathrm{J}} \approx 9 \mathrm{~m}$, and $r=6894$ $\mathrm{km}$ at the flyby, eq. (3) is fully satisfied for Juno. Indeed, $G m_{\mathrm{J}} d_{\mathrm{J}}^{-1}=$ $2.7 \times 10^{-8} \mathrm{~m}^{2} \mathrm{~s}^{-2}$, while $\left|A_{\text {Rin }}\right| r_{\mathrm{J}}=6 \times 10^{-3} \mathrm{~m}^{2} \mathrm{~s}^{-2}$. The magnitude of the putative Rindler-type effect on the Juno's range-rate turns out to be $1.5 \mu \mathrm{m} \mathrm{s}^{-1}$.

It can be noticed that none of the effects considered is able to impact the Juno's range-rate at a $\mathrm{mm} \mathrm{s}^{-1}$ level. Moreover, the smallness of the $1 \mathrm{PN}$ signatures which will likely not be modeled in the data analysis (GM + $J_{2} c^{-2}$ ) should make them practically undetectable. The same holds also for a Rindler-type acceleration $A_{\text {Rin }}$ large enough to explain the Pioneer anomaly. On the one hand, it could not be independently tested with the Moon's motion. Indeed, $G m_{\mathrm{M}} d_{\mathrm{M}}^{-1}=2.8 \times 10^{6} \mathrm{~m}^{2} \mathrm{~s}^{-2},\left|A_{\text {Rin }}\right| r_{\mathrm{M}}=3.3 \times 10^{-1}$ $\mathrm{m}^{2} \mathrm{~s}^{-2}$, so that the condition of eq. (3) would not be satisfied for the Moon. On the other hand, $A_{\text {Rin }}$ could, in principle, certainly affect all the artificial satellites orbiting the Earth along bound trajectories. For, e.g., LARES, it is $[62,63] m_{\mathrm{LR}}=386.8 \mathrm{~kg}, d_{\mathrm{LR}}=36.4 \mathrm{~cm}$, and $r_{\mathrm{LR}}=7828 \mathrm{~km}$; thus $G m_{\mathrm{LR}} d_{\mathrm{LR}}^{-1}=7 \times 10^{-8} \mathrm{~m}^{2} \mathrm{~s}^{-2},\left|A_{\mathrm{Rin}}\right| r_{\mathrm{LR}}=6.8 \times 10^{-3} \mathrm{~m}^{2} \mathrm{~s}^{-2}$, and the condition of eq. (3) is fully satisfied. From [64], it turns out that the perigee precession of LARES is constrained down to $\sigma_{\dot{\omega}_{\mathrm{LR}}}=522$ milliarcseconds per year (mas $\mathrm{yr}^{-1}$ ) level because of unmodeled empirical accelerations in the along-track direction. This rules out the possible existence of an anomalous Rindler-type acceleration for the Earth as large as $A_{\text {Rin }}=8.7 \times 10^{-10} \mathrm{~m}$ $\mathrm{s}^{-2}$ since it would induce an anomalous perigee precession ${ }^{4}$ for LARES as large as $\dot{\omega}_{\text {Rin }}=800$ mas $\mathrm{yr}^{-1}$. From the point of view of the Juno's flyby anomaly, an even smaller magnitude of a putative $A_{\text {Rin }}$, compatible with the LARES bound, would be even more insignificant.

\section{Conclusions}

After its flyby of Earth occurred on 9 October 2013, analyses of the data collected by ESA have been started by NASA/JPL to determine if also Juno, now en route to Jupiter, will exhibit the so-called flyby anomaly which was detected in some of the past flybys of the Galileo, NEAR, and Rosetta spacecraft. An empirical formula proposed to explain the anomalies of such

\footnotetext{
${ }^{3}$ We take the largest dimension of the solar arrays for $d_{\mathrm{J}}$.

${ }^{4}$ The pericenter precession caused by a radial uniform extra-acceleration $A$ is [65-67] $\dot{\omega}=A \sqrt{1-e^{2}} n_{\mathrm{b}}^{-1} a^{-1}$, where $n_{\mathrm{b}}, a, e$ are the satellite's mean motion, semimajor axis, and eccentricity, respectively.
} 

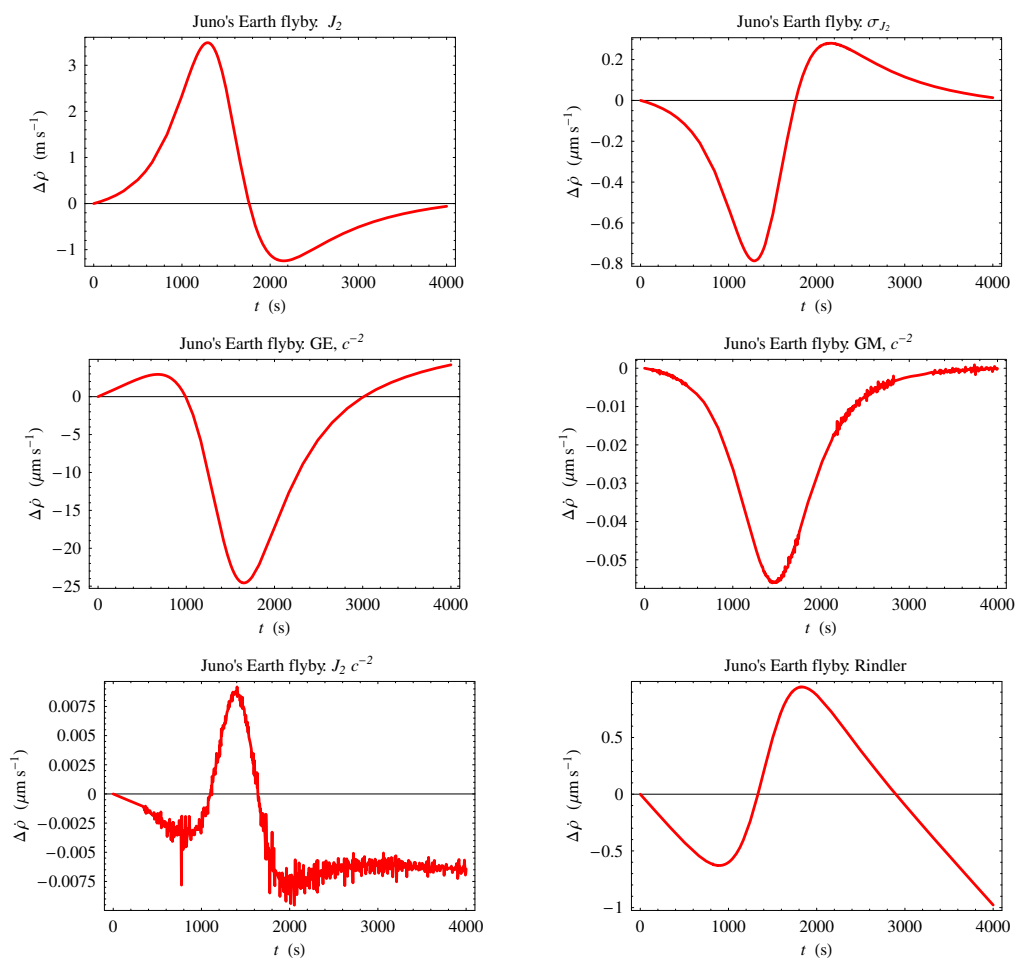

Figure 1: Simulated geocentric range-rate signatures of Juno at the Earth's flyby induced by various standard and non-standard dynamical effects. The units are $\mathrm{m} \mathrm{s}^{-1}$ for the nominal $J_{2}$ signal in the upper left corner, while $\mu \mathrm{m} \mathrm{s}^{-1}$ are used for all the other effects. For the signal induced by the mismodeling in the first even zonal harmonic of the geopotential, displayed in the upper right corner, the conservative value $\sigma_{\bar{C}_{2,0}}=1.09 \times 10^{-10}$ [54], calculated with the approach in [55], is adopted. The Rindler-type curve, placed in the lower right corner, is obtained with the value $A_{\text {Rin }}=8.7 \times 10^{-10}$ $\mathrm{m} \mathrm{s}^{-2}$. 
probes predicts an effect as large as about $6 \mathrm{~mm} \mathrm{~s}^{-1}$ for the Juno's rangerate.

We looked at some dynamical effects which, in principle, may be considered as viable candidates by numerically calculating their effects on the range-rate of Juno at its flyby of Earth. The Earth's quadrupole mass moment $J_{2}$, usually modeled in the data reduction softwares, nominally shifts the Juno's range-rate by a few $\mathrm{m} \mathrm{s}^{-1}$ at the Newtonian level; by conservatively assuming an uncertainty in it of the order of $\approx 10^{-10}$ from the latest global gravity field models, the resulting residual signal reduces down to about $1 \mu \mathrm{m} \mathrm{s}^{-1}$. The general relativistic Schwarzschild-type component of the Earth's gravitational field, which is modeled in the data analyses, causes a nominal range-rate shift of the order of $25 \mu \mathrm{m} \mathrm{s}^{-1}$. The impact of the unmodeled general relativistic Lense-Thirring and $J_{2} c^{-2}$ effects on the Juno's range-rate is at the $0.05-0.01 \mu \mathrm{m} \mathrm{s}^{-1}$ level. A putative Rindler-type radial uniform acceleration of the same magnitude as in the Pioneer anomaly would perturb the Juno's range-rate by $1 \mu \mathrm{m} \mathrm{s}^{-1}$.

If $\mathrm{a} \approx \mathrm{mm} \mathrm{s}^{-1}$ flyby anomaly will finally result also for Juno, it will not be caused by any of the dynamical effects considered in this work. In particular, the unmodeled relativistic signatures will be too small to be

detectable, regardless of any further consideration on the flyby anomaly as a sign of new physics.

\section{References}

[1] S. Matousek, "The Juno New Frontiers mission," Acta Astronautica 61 (Nov., 2007) 932-939.

[2] R. Helled, J. D. Anderson, G. Schubert, and D. J. Stevenson, "Jupiter's moment of inertia: A possible determination by Juno," Icarus 216 (Dec., 2011) 440-448, arXiv:1109.1627 [astro-ph.EP].

[3] S. Clark, "Juno: The spacecraft putting sling theory to the test," (9 October, 2013) . http://www.bbc.com/future/story/ 20131009-spacecraft-tests-sling-theory.

[4] D. Scuka, "Tonights Juno flyby may help unravel a cosmic mystery," (9 October, 2013) . http://blogs.esa.int/rocketscience/2013/ 10/09/ tonights-juno-flyby-may-help-unravel-a-cosmic-mystery/. 
[5] J. D. Anderson, J. F. Jordan, J. K. Campbell, J. E. Ekelund, J. J. Bordi, M. Abrahamson, S. M. Ardalan, and P. F. Thompson, "Juno Earth Flyby as a Sensitive Detector of Anomalous Orbital-Energy Changes," AGU Fall Meeting Abstracts (Dec., 2013) B2187.

[6] H.-J. Busack, "Expected velocity anomaly for the Earth flyby of Juno spacecraft on October 9, 2013," ArXiv e-prints (Sept., 2013), arXiv:1312.1139 [physics.gen-ph].

[7] J. D. Anderson and M. M. Nieto, "Astrometric solar-system anomalies," in IAU Symposium, S. A. Klioner, P. K. Seidelmann, and M. H. Soffel, eds., vol. 261 of IAU Symposium, pp. 189-197. Jan., 2010.

[8] J. D. Anderson, J. K. Campbell, and M. M. Nieto, "The energy transfer process in planetary flybys," New Astronomy 12 (July, 2007) 383-397, astro-ph/0608087.

[9] C. Lämmerzahl and H. Dittus, "The Flyby Anomaly," in The Eleventh Marcel Grossmann Meeting On Recent Developments in Theoretical and Experimental General Relativity, Gravitation and Relativistic Field Theories, H. Kleinert, R. T. Jantzen, and R. Ruffini, eds., pp. 2564-2566. Sept., 2008.

[10] J. D. Anderson, J. K. Campbell, J. E. Ekelund, J. Ellis, and J. F. Jordan, "Anomalous Orbital-Energy Changes Observed during Spacecraft Flybys of Earth," Physical Review Letters 100 no. 9, (Mar., 2008) 091102.

[11] M. M. Nieto and J. D. Anderson, "Earth Flyby Anomalies," Physics Today 62 no. 10, (Oct., 2009) 76-77.

[12] S. G. Turyshev and V. T. Toth, "The Puzzle of the Flyby Anomaly," Space Science Reviews 148 (Dec., 2009) 169-174, arXiv:0907.4184 [gr-qc] .

[13] C. Lämmerzahl, O. Preuss, and H. Dittus, "Is the Physics Within the Solar System Really Understood?," in Lasers, Clocks and Drag-Free Control: Exploration of Relativistic Gravity in Space, H. Dittus, C. Lammerzahl, and S. G. Turyshev, eds., vol. 349 of Astrophysics and Space Science Library, p. 75. 2008. 
[14] B. Rievers and C. Lämmerzahl, "High precision thermal modeling of complex systems with application to the flyby and Pioneer anomaly," Annalen der Physik 523 (June, 2011) 439-449, arXiv: 1104.3985 [gr-qc] .

[15] S. G. Turyshev and V. T. Toth, "The Pioneer Anomaly," Living Reviews in Relativity 13 (Sept., 2010) 4, arXiv:1001.3686 [gr-qc].

[16] J. A. Atchison and M. A. Peck, "Lorentz Accelerations in the Earth Flyby Anomaly," Journal of Guidance Control Dynamics 33 (July, 2010) 1115-1122.

[17] L. Iorio, "The Effect of General Relativity on Hyperbolic Orbits and Its Application to the Flyby Anomaly," Scholarly Research Exchange 2009 (Jan., 2009) 7695, arXiv:0811.3924 [gr-qc].

[18] E. Hackmann and C. Laemmerzahl, "Flyby anomaly and Lense-Thirring effect," in 38th COSPAR Scientific Assembly, vol. 38 of COSPAR Meeting, p. 3845. 2010.

[19] G. U. Varieschi, "Kerr Metric, Geodesic Motion, and Flyby Anomaly in Fourth-Order Conformal Gravity," General Relativity and Gravitation 46 (2014) 1741, arXiv:1401.6503 [gr-qc].

[20] H.-J. Busack, "Simulation of the flyby anomaly by means of an empirical asymmetric gravitational field with definite spatial orientation," ArXiv e-prints (Nov., 2007), arXiv:0711.2781 [physics.gen-ph].

[21] G. G. Nyambuya, "Are Flyby Anomalies an ASTG Phenomenon?," ArXiv e-prints (Mar., 2008), arXiv:0803.1370 [physics.gen-ph].

[22] R. T. Cahill, "Resolving Spacecraft Earth-Flyby Anomalies with Measured Light Speed Anisotropy," Progress in Physics 3 (July, 2008) $9-15$.

[23] K. Svozil, "Microphysical analogues of flyby anomalies," ArXiv e-prints (Apr., 2008), arXiv:0804.2198 [quant-ph].

[24] W. Petry, "A Possible Explanation of Anomalous Earth Flybys," ArXiv e-prints (June, 2008), arXiv:0806.0334 [physics.gen-ph].

[25] M. B. Gerrard and T. J. Sumner, "Earth Flyby and Pioneer Anomalies," ArXiv e-prints (July, 2008), arXiv:0807.3158 [gr-qc]. 
[26] J. P. Mbelek, "Special relativity may account for the spacecraft flyby anomalies," ArXiv e-prints (Sept., 2008), arXiv:0809.1888 [gr-qc].

[27] R. A. Lewis, "Field Theory Model of the Flyby Anomaly," in American Institute of Physics Conference Series, G. A. Robertson, ed., vol. 1103 of American Institute of Physics Conference Series, pp. 226-234. Mar., 2009.

[28] P. A. Murad, "Revisiting Gravitational Anomalies and a Potential Solution," in American Institute of Physics Conference Series, G. A. Robertson, ed., vol. 1103 of American Institute of Physics Conference Series, pp. 302-310. Mar., 2009.

[29] G. Fontana, "Maxwell Formulation of Gravity in the Hyperspace," in American Institute of Physics Conference Series, G. A. Robertson, ed., vol. 1103 of American Institute of Physics Conference Series, pp. 311-316. Mar., 2009.

[30] J. C. Hafele, "Effect of the Earth's Time-Retarded Transverse Gravitational Field on Spacecraft Flybys," ArXiv e-prints (Apr., 2009), arXiv:0904.0383 [physics.gen-ph].

[31] W. Petry, "Non-Prefered Reference Frames and Anomalous Earth Flybys," ArXiv e-prints (Sept., 2009), arXiv:0909.5150 [physics.gen-ph].

[32] H.-J. Busack, "Test for consistence of a flyby anomaly simulation with the observed Doppler residuals for the Messenger flybys of Mercury," ArXiv e-prints (Mar., 2010), arXiv:1006.3555 [physics.gen-ph].

[33] C. Castro, "The Clifford Space Geometry Behind the Pioneer and Flyby Anomalies," International Journal of Modern Physics A 25 (2010) 815-836.

[34] M. Martinis and N. Perković, "On the Gravitational Energy Shift for matter waves," ArXiv e-prints (Apr., 2010), arXiv:1004.0826 [gr-qc].

[35] F. Lassiaille, "Gravitational Model of the Three Elements Theory," International Journal of Modern Physics E 20 (2011) 78-83.

[36] C. K. Raju, "Retarded Gravitation Theory: the Gravitational Velocity Effect in Galactic Rotation Curves, and the Flyby Anomaly," ArXiv e-prints (Feb., 2011), arXiv:1102.2945 [physics.gen-ph]. 
[37] J. C. Hafele, "Effect of the Earth's time-retarded transverse gravitational field on the motion of the Moon," ArXiv e-prints (May, 2011), arXiv:1105.3857 [physics.gen-ph].

[38] W. Petry, "Anomalous Flyby in the Non-Prefered Reference Frame of the Rotating Earth," ArXiv e-prints (Aug., 2011), arXiv:1109.0256 [physics.gen-ph].

[39] C. K. Raju, "Retarded gravitation theory," in American Institute of Physics Conference Series, W. Alves Rodrigues, Jr., R. Kerner, G. O. Pires, and C. Pinheiro, eds., vol. 1483 of American Institute of Physics Conference Series, pp. 260-276. Sept., 2012.

[40] H. K. Tank, "Cumulative-Phase-Alteration of Galactic-Light Passing Through the Cosmic-Microwave-Background: A New Mechanism for Some Observed Spectral-Shifts," Progress in Physics 2 (July, 2012) $39-42$.

[41] M. J. Pinheiro, "The Flyby Anomaly and the Effect of a Topological Torsion Current," ArXiv e-prints (Apr., 2014), arXiv:1404.1101 [physics.space-ph].

[42] L. Acedo, "The flyby anomaly: A case for strong gravitomagnetism ?," Advances in Space Research (2014) . At press.

[43] M. E. McCulloch, "Can the Flyby Anomalies be Explained by a Modification of Inertia," Journal of the British Interplanetary Society 61 (2008) 373-378, arXiv:0712.3022.

[44] M. E. McCulloch, "Modelling the flyby anomalies using a modification of inertia," Monthly Notices of the Royal Astronomical Society $\mathbf{3 8 9}$ (Sept., 2008) L57-L60, arXiv:0806.4159.

[45] S. L. Adler, "Can the flyby anomaly be attributed to earth-bound dark matter?," Physical Review D 79 no. 2, (Jan., 2009) 023505, arXiv:0805.2895.

[46] S. L. Adler, "Spacecraft calorimetry as a test of the dark matter scattering model for flyby anomalies," ArXiv e-prints (Oct., 2009), arXiv:0910.1564 [physics.space-ph].

[47] S. L. Adler, "Modeling the Flyby Anomalies with Dark Matter Scattering," International Journal of Modern Physics A 25 (2010) 4577-4588, arXiv:0908.2414 [astro-ph.EP]. 
[48] S. L. Adler, "Modeling the Flyby Anomalies with Dark Matter Scattering: Update with Additional Data and Further Predictions," International Journal of Modern Physics A 28 (June, 2013) 50074, arXiv:1112.5426 [astro-ph.EP].

[49] O. Bertolami, F. Francisco, P. J. S. Gil, and J. Páramos, "Probing the Flyby Anomaly with the Galileo Constellation," in 3rd International Colloquium. Scientific and Fundamental Aspects of the Galileo Programme, 31 August-2 September 2011, Copenhagen, Denmark. 2011. arXiv:1109.2779 [gr-qc].

[50] O. Bertolami, F. Francisco, P. J. S. Gil, and J. Páramos, "Testing the Flyby Anomaly with the Gnss Constellation," International Journal of Modern Physics D 21 (Apr., 2012) 50035, arXiv:1201.0163 [physics.space-ph].

[51] J. Páramos and G. Hechenblaikner, "Probing the flyby anomaly with the future STE-QUEST mission," Planetary and Space Science $\mathbf{7 9}$ (May, 2013) 76-81, arXiv:1210.7333 [gr-qc].

[52] C. W. F. Everitt, D. B. Debra, B. W. Parkinson, J. P. Turneaure, J. W. Conklin, M. I. Heifetz, G. M. Keiser, A. S. Silbergleit, T. Holmes, J. Kolodziejczak, M. Al-Meshari, J. C. Mester, B. Muhlfelder, V. G. Solomonik, K. Stahl, P. W. Worden, Jr., W. Bencze, S. Buchman, B. Clarke, A. Al-Jadaan, H. Al-Jibreen, J. Li, J. A. Lipa, J. M. Lockhart, B. Al-Suwaidan, M. Taber, and S. Wang, "Gravity Probe B: Final Results of a Space Experiment to Test General Relativity," Physical Review Letters 106 no. 22, (June, 2011) 221101, arXiv:1105.3456 [gr-qc].

[53] W. Heiskanen and H. Moritz, Physical Geodesy. W. H. Freeman, San Francisco, 1967.

[54] L. Iorio, "Constraints from orbital motions around the Earth of the environmental fifth-force hypothesis for the OPERA superluminal neutrino phenomenology," Journal of High Energy Physics 5 (May, 2012) 73, arXiv:1109.6249 [gr-qc].

[55] C. A. Wagner and D. C. McAdoo, "Error calibration of geopotential harmonics in recent and past gravitational fields," Journal of Geodesy 86 (Feb., 2012) 99-108. 
[56] M. Soffel, R. Wirrer, J. Schastok, H. Ruder, and M. Schneider, "Relativistic effects in the motion of artificial satellites. I - The oblateness of the central body," Celestial Mechanics 42 (1988) 81-89.

[57] J. Heimberger, M. Soffel, and H. Ruder, "Relativistic effects in the motion of artificial satellites - The oblateness of the central body II," Celestial Mechanics and Dynamical Astronomy 47 (1990) 205-217.

[58] V. A. Brumberg, Essential Relativistic Celestial Mechanics. Adam Hilger, Bristol, 1991.

[59] D. Grumiller, "Model for Gravity at Large Distances," Physical Review Letters 105 no. 21, (Nov., 2010) 211303, arXiv:1011.3625 [astro-ph.CO].

[60] S. Carloni, D. Grumiller, and F. Preis, "Solar system constraints on Rindler acceleration," Physical Review D 83 no. 12, (June, 2011) 124024, arXiv:1103.0274 [astro-ph.EP].

[61] D. Grumiller and F. Preis, "Rindler Force at Large Distances," International Journal of Modern Physics D 20 (2011) 2761-2766, arXiv:1107.2373 [astro-ph.CO].

[62] A. Paolozzi, I. Ciufolini, and C. Vendittozzi, "Engineering and scientific aspects of LARES satellite," Acta Astronautica 69 (Aug., 2011) $127-134$.

[63] A. Paolozzi and I. Ciufolini, "LARES successfully launched in orbit: Satellite and mission description," Acta Astronautica 91 (Oct., 2013) 313-321, arXiv:1305.6823 [astro-ph.IM].

[64] G. Renzetti, "First results from LARES: An analysis," New Astronomy 23 (Oct., 2013) 63-66.

[65] L. Iorio and G. Giudice, "What do the orbital motions of the outer planets of the Solar System tell us about the Pioneer anomaly?," New Astronomy 11 (July, 2006) 600-607, gr-qc/0601055.

[66] R. H. Sanders, "Solar system constraints on multifield theories of modified dynamics," Monthly Notices of the Royal Astronomical Society 370 (Aug., 2006) 1519-1528, astro-ph/0602161.

[67] M. Sereno and P. Jetzer, "Dark matter versus modifications of the gravitational inverse-square law: results from planetary motion in the 
Solar system," Monthly Notices of the Royal Astronomical Society 371

(Sept., 2006) 626-632, astro-ph/0606197. 